Claremont Colleges

Scholarship@Claremont

WM Keck Science Faculty Papers

W.M. Keck Science Department

11-1-1989

\title{
Complex Motor Dynamics and Control in Multi- looped Negative Feedback Systems
}

Anne Beuter

University of Quebec at Montreal

John Milton

Claremont McKenna College; Pitzer College; Scripps College

Christiane Labrie

Leon Glass

McGill University

\section{Recommended Citation}

Beuter, A., J. Milton, C. Labrie, L. Glass. "Complex Motor Dynamics and Control in Multilooped Negative Feedback Systems." Conference Proceedings for the IEEE International Conference on Systems, Man and Cybernetics, vol. 3 (14-17 November 1989): 899-902. DOI: 10.1109/ICSMC.1989.71426 accepted for inclusion in WM Keck Science Faculty Papers by an authorized administrator of Scholarship @ Claremont. For more information, please contact scholarship@cuc.claremont.edu. 
Abstract: Experiments were performed in normal and Parkinsonian subjects who were asked to maintain a constant finger position using a time-delayed visual feedback. The finger position showed complex dynamics, with characteristic differences between normal and Parkinsonian subjects. Here we discuss some of the theoretical issues that must be resolved in order to understand the mechanisms that underly the generation of these complex signals.

\section{INTRODUCTION}

Goal directed movements comprise most of human activity. These movements form a vast repertoire that increases with development and learning and which is continuously modified by environmental and behavioral constraints. In health, voluntary movements and gestures are constantly modified in order to optimize certain criteria, e.g., energy, speed, gracefulness. However, in disease motor dysfunction is often characterized by the presence of uncontrolled oscillations some of which are very complex, e.g., tremors, chorea, athetosis. The regulation of movement involves the cooperation of multiple feedback loops operating at multiple levels in the peripheral and central nervous system. Each feedback loop has its own characteristic time delay and gain. Models of multiple delayed feedback loops are capable of generating complex dynamics (1) and thus these models offer a possible explanation for the complex movements observed in health and disease.

Even the "simplest" movements, such as pointing a finger, involve most of the structures of the central nervous system. In such a task, visual input is required to attend to the target and to estimate the discrepancy, or error, between the position of the finger and the position of the target. This error gives rise to a signal which is fed back to the mechanisms controlling finger movement and is used to adjust finger position thus minimizing the error. This cycle between percelved error and movement production forms a closed feedback loop. However, this feedback likely involves several control mechanisms (Figure 1). Afferent signals arising from the proprioceptors and the retina

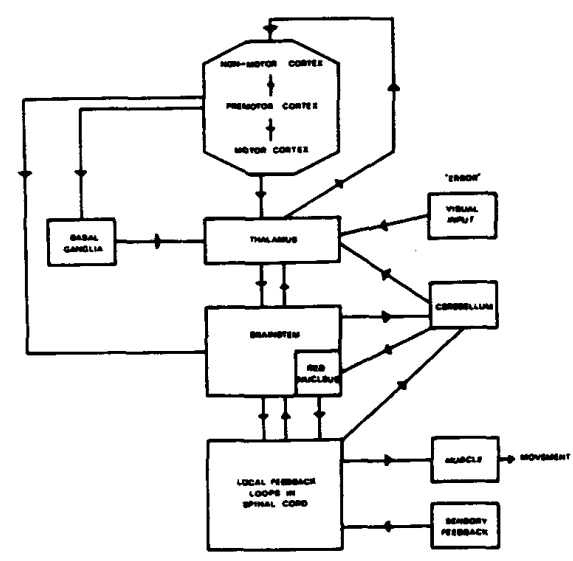

Figure 1: iNeuroanatomical model of the multiple movement of the index finger.

travel by separate pathways to the cortex via separate nuclei of the thalamus and the basal ganglia. The likely first sites of interaction of this afferent information for the task of altering finger position are the association areas of the cortex, 1.e., the parieto-occipital lobes. Descending information to the spinal cord and the finger via the corticospinal and rubrospinal tracts and ascending information from vision (i.e., superior colliculus) and limb movements (i.e., spinocerebellar tract) are modulated by the cerebellum.

One of the advantages of studying visuallyguided movement tasks, 1.e., tracking, is the ease by which it is possible to modify the latency and/or gain of the visual feedback loop. Here we show that complex oscillations in index finger displacement can be produced by introduction of a visual delay into a simple tracking task. The extent to which these observations can be interpreted in the context of models of multi-looped feedback networks is discussed. The results are described more fully in 1-3. An alternative approach to the study of finger movements is being developed by Kelso and co-workers (4).

CH2809-2/89/0000-0899\$1.00 01989 IEEE 


\section{METHODS}

The experimental design is described in detail elsewhere (1-3). Briefly, subjects are required to adjust the position of their index finger to match the position of a target. However, the subjects are not able to see the position of their index finger in relation to the target. Instead they look at an oscilloscope screen on which two horizontal lines are displayed. One Ine is stationary and corresponds to the target. The other is controlled by the index finger at the metacarpo-phalangeal joint as detected by a microdisplacement transducer (LVDT). The subjects are asked to match the two horizontal lines as closely as possible. A variable time delay is introduced by inserting an analog delay 1 ine (bucket brigade type device) between the transducer and the oscilloscope. Thus, by viewing the oscilloscope screen the subjects are only able to judge the position of their index finger relative to the target at some time in the past. In addition, the feedback gain can be increased by amplifying finger movements so that relatively larger displacements are seen on the oscilloscope screen. In these experiments, a finger displacement of $1 \mathrm{~mm}$ corresponds to a displacement of about $16 \mathrm{~mm}$ on the oscilloscope screen.

\section{RESULTS}

The effect of introducing a time delay in the visuo-motor feedback loop is illustrated in Figures 2 and 3 . When no time delay is inserted (Figure 2) the oscillations recorded correspond to tremor. With a time delay of $1500 \mathrm{~ms}$, irregular fluctuations in finger displacements occur which are not present at zero delay (Figure 3). The average inter-peak interval of the regular oscillation increases continuously with the time delay and is always between two and four times the delay. These slow oscillations appear intermittently during the $60 \mathrm{~s}$ record.

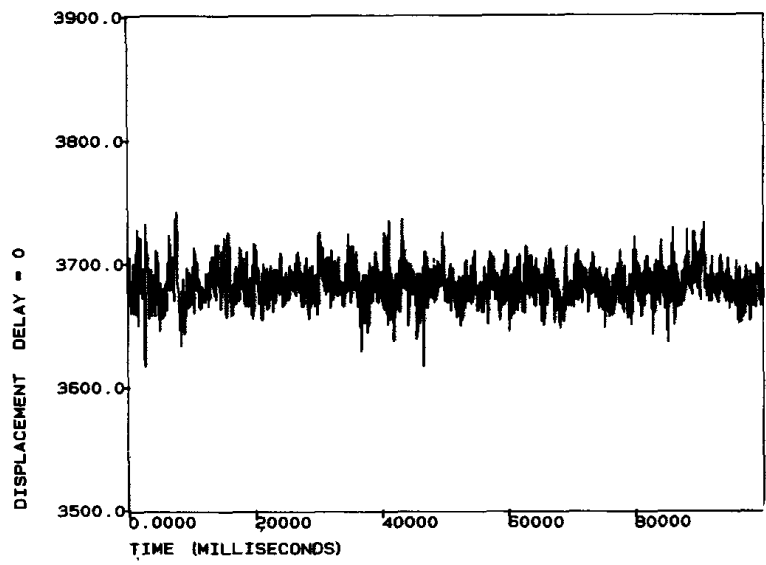

Figure 2: Finger oscillations with no time delay. $50 \mathrm{AD}$ units correspond to approximately $1 \mathrm{~mm}$.

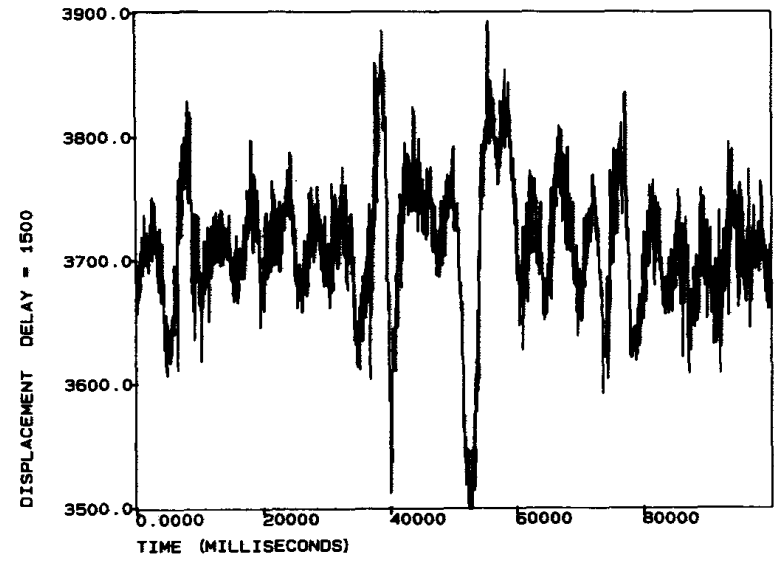

Figure 3: Finger oscillations with a time delay of $1500 \mathrm{~ms}$.

One interesting phenomenon observed in a patient with Parkinson's disease (basal ganglia lesions) is an abrupt change in the qualitative nature of the time series for finger displacement (Irirure 4 ). This phenomenon has been reported

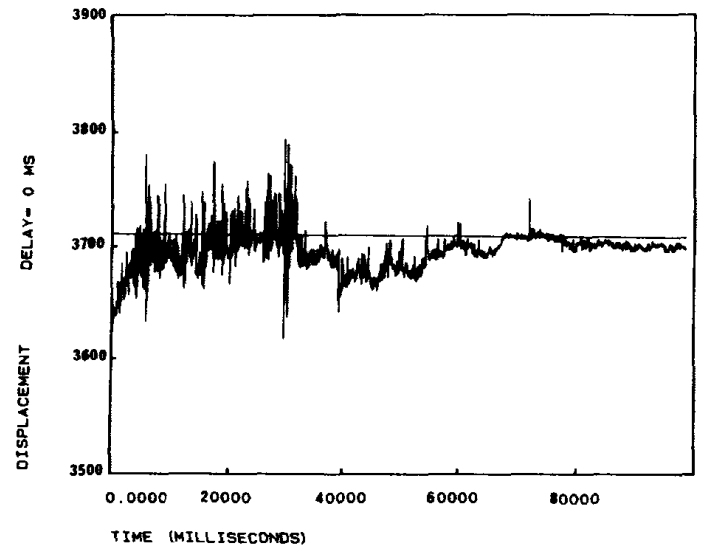

Figure 4: Transition in finger oscillations for a patient with Parksinson's disease

before (5) and consists of an abrupt change in both the amplitude and frequency of the movement.

\section{DISCUSSION}

The visually guided motor task described in this experiment is very simple. However, the observed time series of finger displacement generated by this task are extraordinarily complicated. Here we briefly discuss some of the theoretical issues that must be resolved in order to understand the mechanisms that underly the generation of these complex signals.

It is well known that negative feedback 
systems with single control loops can be destabilized by increasing the gain and/or delay to produce regular oscillations with periods between two and four times the delay (6). Although low frequency oscillations with periods of this magnitude are observed for most subjects, these oscillations do not have a regular waveform over the entire $60 \mathrm{~s}$ record. Indeed the striking feature of the temporal record is not the stability of the low frequency oscillations, but rather its fluctuating and aperiodic nature. There are many mechanisms that may contribute to the generation of time signals of this complexity. These include: (i) the interactions between the multiple feedback loops; (ii) the interactions between feedforward and feedback loops; and (iii) the possibility that parameters in the feedback system are not constant, but may be regulated by the state of the system, or fluctuate due to random inputs to the control system.

Since multiple control loops are involved in the control of movement (Figure 1), it is possible that the complex time series we observe reflect the dynamics of multi-loop feedback systems. Previous workers (7) emphasized that multiple feedbacks would tend to stabilize feedback systems. This is not always the case. In a recent paper (1) we found that multiple negative feedback could, in some circumstances, lead to deterministic chaotic dynamics in which there was aperiodic dynamics with sensitive dependence on the initial conditions. Thus, multiple negative feedbacks can lead to a destabilization of the dynamics and to complex temporal fluctuations. However, recent studies (8) have shown that although chaotic dynamics can be found in multiple negative feedback systems, this is a comparatively rare phenomenon, at least in the specialized systems that were examined. Thus, it is unlikely that the dynamics of deterministic models of multi-loop negative feedback systems can completely account for these observations.

One factor that should be considered in theoretical models of motor control is the role of feedforward control. The organization of goa1directed movements generally can be described functionally as a continuum. At one extreme, a movement to a target may be continuously adjusted using feedback mechanisms. At the other extreme, a movement may involve a series of preprogrammed commands unfolding in a feedforward mode with few opportunities of current modification by proprioceptive or other feedback mechanisms. Mathematically, such preprogrammed commands can be incorporated into theoretical models by computing the position at some future time and modifying muscular activity based on the deviation of the limb tip from its desired location. Thus, both feedback and feedforward loops must be considered in theoretical models of motor control (9).

Finally the role of fluctuations in the gain, delay and other parameters describing the feedback loops, in shaping the observed dynamics must be considered. One possibility is that these parameters are some function of the current state of the system. For example, control mechanisms with state-dependent delays have been recently considered in other contexts (10). Alternatively there may be stochastic fluctuations in some of these parameters since they reflect underlying neurophysiological and molecular processes.

\section{CONCLUDING REMARKS}

Further experimental and/or clinical studies are needed to compliment theoretical approaches. One approach is to study patients with known lesions in neural centers directly involved in motor control (Figure 1). Indeed by careful patient selection it is possible to identify patient subgroups with well localized lesions. For example, we have shown that lesions in the basal ganglia that occur in patients with Parkinson's disease do not greatly affect their performance in this tracking task if their parkinsonian tremor is of relatively low amplitude (11). There is a need to extend these studies in order to examine patients with cerebellar disease and other neurological disorders. Such a strategy is directed at identifying those feedback loops which are critical for this motor task.

An integration of approaches from many disciplines including control theory, nonlinear dynamics and neurology will be needed to understand the complex time series generated in the neuromotor system regulating finger position under feedback control. We may then become able to address questions such as the following: Do motor commands involve different brain circuits for feedback and feedforward modes? What are the mechanisms responsible for dynamic switching. between one mode and the other? What are the interactions between peripheral, subcortical and intracortical loops during goal-directed movement?

Acknowledgements: This work was partly supported by Natural Sciences and Engineering Research Council (Canada) and Fonds pour 1a Formation de Chercheurs et 1 'Alde a la Recherche (Quebec) through the University of Quebec and McGill University.

\section{REFERENCES}

1. L. Glass, A. Beuter and D. Larocque, "Time delays, oscillations and chaos in physiological control systems," MATH. BIOSCI. Vo1. 90, pp. 111-125: 1988 .

2. A. Beuter, D. Larocque and L. Glass, "Complex oscillations in a human motor system," $\mathrm{J}$. MOTOR BEH. (in press).

3. J.G. Milton, A. Longtin, A. Beuter, M.C. Mackey and L. Glass, "Complex dynamics and bifurcations in neurology," J. THEORET . BIOL. Vol. 138, pp. 129-147 : 1989.

4. G. Schoner and J.A.S. Kelso, "Synergetit theory of environmentally-specified and learned patterns of movement coordination. Parts I and II," BIOL. CYBERN. Vol. 58, pp. 71-80;81-89: 1988 .

5. V.S. Gurfinkel and S.M. Osovets, "Mechanism of generation of oscillations in the tremor 
form of parkinsonism," BIOFIZIKA Val. 18, Pp. 731-738: 1973 .

6. L. Glass and M.C. Mackey. From clocks to chaos: the rhythms of life. Princeton University Press, Princeton: 1988.

7. M.N. Oguztorelli and R.B. Stein, "The effects of multiple reflex pathways on the oscillations in neuro-muscular systems," J. MATH. BIOL. Vol. 3, pp. 87-101 : 1976.

8. L. Glass and C.P. Malta, "Chaos in multilooped negative feedback systems," (In review).

9. H. Hemani and B.T. Stokes, "A qualitative discussion of mechanisms of feedback and feedforward in the control of locomotion," IEEE TRANS. BIOMED. ENG. Vo1. BME-30, pp. 681-691 : 1983 .

10. M.C. Mackey, "Commodity price fluctuations: Price dependent delays and non-linearities as explanatory factors," J. ECON. THEORY (in press).

11. A. Beuter, J.G. Mi1ton, C. Labrie, L. Glass and S. Gauthier, "Effects of delayed visual feedback on motor control in Parkinson's disease," (in review). 